Aus der Hautklinik der Universität in Bonn.

(Direktor: Prof. E. Hoffmann.)

\title{
Zur Wirkung reiner Silbersalvarsankuren bei frischer Syphilis und syphilitischer Nephrose.
}

\section{Von Dr. E. Zurhelle.}

In dem Maße, wie die Therapia magna sterilisans Ehrlichs auf chemotherapeutischem Wege sich als undurchführbar erwies, ward im Laufe der letzten Jalıre, gegrüıdet auff zahllose klinische Beobachtungen und Erwägungen und jahrhundertelange Erfahrung der Syphilisbehandlung, das System einer kombinierten Hg-Salvarsanbehandlung ausgebaut.

Diese Kombination glaubte Kolle durch das Silbersalvarsan erfolgreich ersetzen zu können; als ein noch stärkeres Mittel sollte es die Kombination mit $\mathrm{Hg}$-Behandlung überflüssig machen.

Bereits Ehrlich hatte uns Salvarsanpräparate in die Hand gegeben mit so starker spirochätozider Wirkung, daB nur die stellenweise unerreichbare und geschützte Lagerung der Spirochäten im Gewebe den vollen Erfolg vereiteln konnte; daher die Behandlung mittels kombinierter Kur zur besseren Beeinflussung der noch übrig rebliebenen Krankheitserreger. Konnte doch $\mathrm{F}$ is $\mathrm{Cl}_{1} 1^{1}$ ) nach hohen Gabeı Neosalvarsan (von 0,75 bzw. 0,9 in kurzen Abständen) beim Primäraffekt immer noch Spirochäten im Ausstrich, Schnitt oder Tierversuch nachweisen. Bei dem an unserer Klinik iibliclien KurmaB (Salvarsannatrium und Altsalvarsan) hatten diese Versuche allerdings stets negativen Erfolg (vgl. Ha bermann, Derm. Zschr. 22, H. 7),

Es mußte die Gefalır bestehen, daß diese nicht oder nur un. vollkommen abgetöteten Spirochäten infolge der durch den chemotherapeutischen Erfolg beeinträchtigten natürlichen Immunitätsverhältnisse des Körpers an dell versteckten Stellen erst recht sich vermehren und zu Neurorezidiven, vielleicht auch zu ungünstiger Wirkung auf das Schwinden der Wa.R. führen könnten. Das konnte auch für das in geringerer Dosis gebräuchliche Silbersalvarsan gelten, selbst wenn seine beim Kaninchen gesteigerte chemotherapeutische Wirksamkeit auch beim Menschen sich voll bewährte.

Tatsächlich sind Neurorezidive nach Silbersalvarsan nicht ganz selten beobachtet worden, und auch ein verlangsamtes Schwinden der Wa.R. ist voll Kerl, Fabry, Knopf und Sinn, Schönfeld und Birnbaum angegcben worden. In der neuesten Arbeit aus der Jadassohnschen Klinik berichtet auch Wi $W^{2} \mathrm{r}^{\mathrm{a}}$ ) über elf Fälle von Sero- bzww. Neurorezidiven. Auch $S$ tühme ${ }^{3}$ ) berichtet bei 77 teils rein mit Silbersalvarsan, teils in Kombination mit Sulfoxylat behandelten Fälleı von Lues II über 24 Fälle, die am SchluB der Kur noch stark positiv, 16 Fälle, die noch schwach positiv re. agierten, denen sich melırere Serorezidive, 5 klinische Rezidive (zum Teil mit Roseola) und 2 Neurorezidive hinzugeselltelı.

Immerhin sprach sich die bei weitem überwiegende Mehrzahl der Dermatologen so günstig über die Wirkung der reinen Silbersalvarsantherapie aus, daß skeptischere Stimmen lange Zeit finst ganz in den Hintergrund traten.

Ohgleich die Beurteilung an unserer Klinik. wie aus den Arbeiten yon Sinn und Knopf hervorgeht, sehr zurückhaltend war, habe ich Versuche mit reiner Silbersalvarsantherapie, speziell auch auf die guten Erfahrungen Galewskys hin, fortgesetzt. Die Dosierung war entsprechend $G$ alewsky äußerst vorsichtig. Jedoch erwies es sich nicht als notwelldig, unter die Dosis von 0,1 (oder 0,15 ) $\mathrm{zu}$ Beginn hinunterzugehen. Irgendwelche Schädigungen wurden auch dabei nicht beobachtet; anderseits wurde eine exzitierende, die Spiro. chaete pallida mobilisierende Wirkung eher vermieden.

$\mathrm{Zu}$ Neurorezidiven kam es bei dell neuerdings in Behandlung genommenen Patienten niclit, demgegenüber konnte ich die Klagen über schlechte Beeinflussung der Wa.R. ergänzen.

Als Beleg dienen folgende Krankengeschichten:

F all 1. P. S., 47jähriger, verheirateter Mann. Aufnahme 20. IV. 1920. L. C. extra muros Sommer 1918. Einige Wochen später Ge. schwüre auf der Bauchhaut, drei Finger breit oberhalb des Gliedansatzes. September 1919 Drüsenschwellung in rechter Leiste, gleichzeitig kleines Geschwür am Glied und Vorhautverengerung. Damals ca. drei Wochen lang auswärts mit ( $\mathrm{Hg}$-)Injektionen in die Glutäen behandelt. Diagnose: Lues Il (Papeln am After, an der Vorhaut und Eichel, Plaques muqueuses der Unterlippe, Scleradenitis multiplex). Behandlung: 20. IV. I. Silbersalvarsannatriuminjelktion 0,$1 ; 23$. IV II. S.S. 0,$15 ; 26$. IV. III. S.S. 0,$2 ; 29$. IV. IV. S.S. 0,$25 ;$ weiter in dreitägigen Abständen je 0,25. Wa.R. am 28. V. (bei XIl. Inj.) noch $3-4$, nach 2,45 S.S. innerhalb von 35 Tagell.

F all 2. C. B., 21jähriger Mann. L. C. November oder Dezember 1918. Aufnahme 30. III. 1920. Diagnose: Lues II (Papel der Oberlippe unter dem linken Nasenloch, Papeln der Glans). Behandlung: 31. Ill. I. S S. 0,1 : 3. IV. Il. S.S. 0,$1 ; 6$. IV. III. S.S. 0.15; 9. IV. IV. S.S. 0,$2 ; 12$ IV. V.S.S. 0,25; weiter in dreitägigen Abständen je 0,25 . Am 4. V. 1920 Wa.R.: 4 (bei XII. S.S.-Inj.), nach 2,3 S.S. innerhalb von 32 Tagen.

Fall 3. H.K., 24jähriger Mann. Aufnahme 1. V. 1920. Patient wurde bereits im Januar 1920 wegen verhärteter Ulzeration im

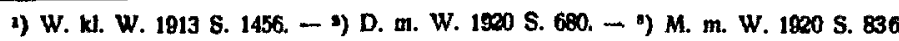


Sulcus coronarius von Homōopathen auf Lues behandelt (6 Injek. tionen in die GesäBmuskulatur). L. C. Anfang April 1020. Diagnose: Lues II. (Makulopapulöses Exanthem, Polyskleradenitis.) Behandlung 2. V. 1920 I. S.S. 0,$1 ; 5$. V. II. S.S. 0.1 ; 8. V. III. S.S. 0,2 ; 11 . V. IV. S.S. 0,25 ; weiter in dreitägigen Abständen je 0,25 , Wa.R. be IX. Inj. am 28. V. noch 4, bei XI. Inj. am 3. VI. 1-2, nach 1,9 S.S. innerhalb von 29 Tagen.

F all 4. M. S., 26jähriger Mann. Aufuahme 19. III. 1920. L. C. nicht mit Siclıerheit zu eruieren. Seit 7 Wochen entzündliche Phinose und seit ungefähr derselben Zeit Ausschlag am Körper. Diagnose Lues II. (Papulöses Exanthem, Polyskleradenitis.) Belaaudlung: 20. III I. S.S. 0,05; 23. III. II. S.S. 0,1; 25. III. III. S.S. 0,15; 29. III. IV. S.S. 0,2 1. IV. V. S.S. 0,25; weiter in dreitägigen Abständen je 0,25. Wa.R am 24. IV. 1920 (bei XII. Inj.) noch 3-4, nach 2,1 S.S. innerhalb von 33 Tagen.

F all 5. P. R., 19jähriger Mann. Aufnahme 19. Il. 1920. L. C. 5-6 resp. 9 Wochen vor der Aufnahme, seit drei Wochen entzündliche Phimose. Diagnose: Lues II. (Roseola, Polyskleradenitis.) Behandlung: 20 . II. I. S.S. 0,$05 ; 23$. II. II. S.S. 0,$05 ; 26$. II. III. S.S. 0,15 29. II. IV. S S. 0,$2 ; 4$. III. V. S.S. 0,$25 ; 8$. III. IV. S.S. 0,$3 ; 11$. III VII. S.S. 0,$25 ; 14$. III. VIII. S.S. 0,$25 ; 19$. III. IX. S.S. 0,25 Wa.R. 4 26. III. X. S.S. 0,25 , Wa.R. $1-2 ; 30$. III. XI. S.S. 0,25 , Wa.R. $1-2$; 6. IV. XII. S.S. 0,25 Wa.R. mit einem Extrakt 3-4, mit einem Extrakt negativ, nach 2,25 S.S. innerhalb von 39 Tagen.

Fall 6. Kl., 26jähriger Manu. Aufnahme 5. V. 1920. Patient bemerkte seit 5 Wochen ein Geschwür am Glied. Seit 5 Tagen Ausschlag am Körper und Rachenentzündung. Infektionsmöglichkei in denl letzten Monaten häufiger. Diagnose: Lues II. (Zwei Primäraffekte der Kranzfurche, kleinpapulöses Exanthem am ganzen Körper Angina specifica, Polyskleradenitis.) Behandlung: 6. V. 1920 I. S.S. 0,1 9. V. II. S.S. 0,$1 ; 12$. V. III. S.S. 0,$15 ; 15$. V. IV. S.S. 0,$2 ; 18$. V V. S.S. 0,25 ; weiter in dreitägigen Abständen je S.S. 0,25. Wa.R. am 12. VI. (bei XIl. Inj.) noch 2-3; am 16. Vl. (bel XIII. Inj.) mit einem Extrakt 4, mit einem Extrakt negativ, nach 2,3 S.S. innerhalb von 34 Tagen.

Wenn man auch in den Fällen 1 bis 3 einwenden kann, daß es sich um länger zurückliegende Infektionen handelt, so ist dies bei den Fällen 4 bis 6 doch kaum der Fall.

Ganz abgesehen von der theoretischen Frage, wodurch denn diese hartnäckige Wa.R. erhalten bleibt, sind diese Beispiele auch von eminent praktischem Interesse.

Wenn bei Lues II nach 12 Injektionen Silbersalvarsan die Wa.R positiv geblieben ist, beginnt die zweite oder Sicherheitskur nach $11 / 2$ bis 2 Monater, falls die $W a . R$. nicht in der Zwischenzeit nach Ablauf der ersten Kur noch negativ geworden ist, im seropositiven Stadium, kann also kaum als Sicherheitskur gerechuet sondern muB als erste Kur eines Serorezidivs angesehen werden, der eine zweite Kur als endgültige Sicherheitskur folgen müßte. Es sei denn, daß man sich entschlösse, solange mit Silbersalvarsan über die 12. Injektion hinaus weiter zu behandeln, bis die Wa.R. negativ geworden ist.

Diese Gedankengäıge veranlaBten uns, die reine Silbersalvarsantherapie bei Lues II einzuschränken. Um so mehr, als auch Bruck und So $\mathrm{mmer}$ ') bei 17 rein mit Silbersalvarsan behandelten Fällen von Lues II in serologischer Beziehung 10 MiBerfolgen gegenüber nur 7 Erfolge $z u$ verzeichnen hatten. Von ihren 10 als Mißerfolg bezeichneten Fällen waren 5 auch nach der Kur positiv geblieben. Auch aus der Jadassohnschen Klinik hat Wiener (l. c.) vor kurzem die reine Silbersalvarsanbehandlung in erster Linie für Lues I empfohlen. Immerhin konnte bei Lues I seropositiva Loe w $y^{2}$ ) aus unserer Klinik unter 14 Fällen, trotz ausgiebigster Behandlung, leider über 3 Rezidive berichten. Die St ühm e rsche SchluBfolgerung, daß bei Silbersalvarsanbehandlung die gleichzeitige Anwendung von $\mathrm{Hg}$ bei primärer Syphilis mit noch negativer Wa.R. entbehrlich sei, ist also auch nach unseren Erfahrungen auf dieses Stadium zu beschränken.

Trotzdem behielten wir die Indikation zur reinen Silbersalvarsantherapie mit Rūcksicht auf den großen Vorteil einer $\mathrm{Hg}$-losen $\mathrm{Be}$ handlung ständig im Auge und glaubten, bei syphilitischer $\mathrm{Nephrose}$ eine Indikation zu finden, die bisher kaum beachtet, ein gewisses Interesse beansprucht.

Veranlassung dazu bot folgender Krankheitsfall

H. H., 70(!)jähriger Mann, wurde von der Medizinischen Klinik, wo er seit 1. V. 1920 wegen dreitāgiger Pneumonie (Fieber bis $39,7^{\circ}$ ) behandelt worden war, am 11. V. 1920 in die Hautklinik verlegt wegen Lues II. Der Ürin enthielt am 2. V. $5 \%$ EiweiB, im Sediment fanden sich Leukozyten, Erythrozyten, Epithelzylinder. Wa.R. am 3. V. positiv. Blutdruck (nach Recklingliausen) am 7. V. $121 / 50 \mathrm{~mm} \mathrm{Hg}$, am 9. V. $134 / 60 \mathrm{~mm} \mathrm{Hg}$. Eiwe iB gehalt a m 7. V. $20 \% \%$. Am 10. V. traten starke Oedeme beider Beine auf, Anasarka, Aszites (?). Die Diagnose der Medizinischen Klinik lautete: Chronische Nephritis mit luetischer (?) Nephrose. Von früheren Kiankheiten wurde Lungenentzündung und Rippenfellentzündung vor 20 Jahren angegeben. Sonst will Patient nie ernstlich krank, insbesondere nie geschlechtskrank gewesen sein. L. C. angeblich Ende März 1920. Befund am 11. V. 1920 (bei der Verlegung): Alter Mann in gutem Ernährungszustande. Starkes Oedem beider Beine. des Penis und Skrotums; Anasarka am Unterleib, in den Seiten und

1) Derm. Zschr. 1920 8. 129. - ") Dlas. Bonn 1920. am Rücken. Es besteht heute deutlich nachweisbarer Aszites. Die Stimme ist heiser. Mäßiger Husten mit wenig zähschleimig-eitrigem Auswurf. Die Lungengrenzen sind tiefstehend, wegen Anasarka nicht deutlich abgrenzbar, schlecht verschieblich. Der Klopfschall voll. Ueberall Giemen. Der zweite Ton über der Aorta betont. Puls regelmäßig, kräftig, 80 in der Minute. Die ödematös geschivollene Vorhaut zeigt am inneren Blatt unten ein erbsengroßes Geschwür von schinkelnroter Farbc mit haselnußdicker Induration in der Um. gebung. Die Inguinaldrusen sind infolge des Oedems nicht palpabel, die Maxillardrüsen beiderseits haselnußgroB, die Nuchaldrüsen erbsengroß, die Axillardrüsen von gleicher Größe, die Kubitaldrüsen sind nicht deutlich zu fühlen. Am Rumpf und an den Oberarmen eine ganz schwach ausgeprägte, mittelgroßfleckige Roseola, am Skrotum einige in Abheilung begriffene Papeln, in denen Spirochäten sich nicht mehr nachweisen ließen. Mund und After o. B. Reflexe regel. recht. Wa.R. ++++ . 12. V. Urinmenge von 12 Uhr mittags des 11. V. bis heute $9 \mathrm{Uhr}$ vormittags $600 \mathrm{ccm}$. Spez. Gew. 1020. Nach Esbach ca. 12\% Eiweili. Im Urinsediment Leukozyten, Erythrozyten, Bruchstücke granulierter und hyaliner Zylinder mit einzelnen Partikelchen doppelbrechender Substanzen, vereinzelte Epithelien. Diagnose: Lues II. (Roseola, luetische Nephrose, Polyskleradenitis.) B e handIung: Zunächst reine Silbersalvarsantherapie, kochsalzarme Diät, Beschränkung der Flüssigkeitszufuhr, Bettruhe. I. Inj. von Silbersalvarsannatrium 0,1, Wa.R. 4

13. V. Urinmenge von 9 Uhr bis 9 Uhr morgens $660 \mathrm{ccm}$. Spez. Gew. 1020. Esbach 6\%. Eine weitere Verfolgung der doppelbrechenden Substanzen unterblieb aus äußeren Gründen. Gegen Abend Temperatursteigerung auf 38,0 . Wadenumfang $13 \mathrm{~cm}$ unter dem unteren Kniescheibenrande beiderseits $39,5 \mathrm{~cm}$.

14. V. Urinmenge $900 \mathrm{ccm}$. Spez. Gew. 1025. Esbach $6 \%$

15. V. II. S.S. 0,1. Urinmenge $900 \mathrm{ccm}$. Spez. Gew. 1022. Esb. $3 \%$ Der Wadenumfang hat beiderseits um $11 / 2 \mathrm{~cm}$ abgenommen. 16. V. Urinmenge $450 \mathrm{ccm}$. Spez. Gew. 1018. Esb. 2\%. Der Wadenumfang hat wieder zugenommen und beträgt heute $39 \mathrm{~cm}$.

Auch das Oedem des Skrotums hat sich erheblich vermehrt.

17. V. Urinmenge $2000 \mathrm{ccm}$. Spez. Gew. 1012. Esb. $3 / 4 \%$

18. V. Urinmenge $2450 \mathrm{ccm}$. Spez. Gew. 1012. Est. $1 / 4 \%$ I. S.S. 0,15 , Wa.R. 4 .

19. V. Urinmenge $2200 \mathrm{ccm}$. Spez. Gew 1010. Esb. Spur. Die Oedeme haben überall abgenommen, der Wadenumfang beträgt Deiderseits $36 \mathrm{~cm}$

20. V. Urinmenge $2200 \mathrm{ccm}$. 'Spez. Gew. 1012. Esb.: Spur. 21. V. Urinmenge $2400 \mathrm{ccm}$. Spez. Gew. 1010. Esb.: Spur. S. $0,2, W_{a} R, 4$

22. V. Urinmenge $2600 \mathrm{ccm}$. Spez. Gew. 1012. EiweiB ist nicht mehr nachzuweisen (und tritt auch in der Folge nicht mehr auf). Im Urinsediment keine pathologischen Bestandteile mehr.

23. V. V. S.S. 0,2, Wa.R. 4 .

24 . V. Wadenumfang rechts 35,5 , links $34 \mathrm{~cm}$.

25. V. Das spez. Gew. ist bis auf 1005 gesunken

26. V. VI. S.S. 0,25, Wa.R. 4. Die Oedeme sind fast vollständig geschwunden; Wadenumfang rechts und links $33 \mathrm{~cm}$. Beginn einer Imierkur mit $21 / 2$ g

29. V. VII. S.S. 0,25. Wa.R. 4. Patient stelit stundenweise auf.

1. VI. VIII. S.S. 0,25 .

4. VI. IX. S.S. 0,25, Wa.R. 4 .

7. Vl. X. S.S. 0,25, Wa.R. 4. Patient steht den ganzen Tag auf

10. VI. XI. S.S. 0,25

13. VI. XII. S.S. 0,25, Wa.R. 4. Patient besucht den Garten.

16. VI. Blutdruck (nach Recklinghausen) in Medizinischer Klinik $00 / 44 \mathrm{~mm} \mathrm{Hg}$

18. VI. Xill. S.S.natrium 0,45, Wa.R. 4

19. VI. Patient schmiert von heute ab mit $5 \mathrm{~g}$ täglich.

23. VI. XIV. S.S. 0,45. Wa.R. mit einem Extrakt negativ, mit einem Extrakt 2

28. VI. XV. S.S. 0,45."Wa.R. mit einem Extrakt negativ, bei einem Extrakt leichte Hemmung.

30. Vl. Wa.R. mit beiden Extrakten negativ.

2. VII. Patient wird mit der Weisung entlassen, sich nach 6 Wochen zur Sicherheitskur wieder einzufinden. Er hat im ganzen 30 Einreibungen gemacht (20 mit je $21 / 2 \mathrm{~g}$ und 10 mit je $5 \mathrm{~g}$ ).

Die Krankengeschichte ist in verschiedenter Hinsicht lehrreich. Zunächst konnte durch erfolgreiche antisyphilitische Behandlung die Diagnose "chronische Nephritis mit luetischer Nephrose“ in syphilitische Nephrose verifiziert werden. Der am 9. V. 1920 festgestellte erhöhte Blutdruck dürfte deshalb weniger in dem Nierenleiden als in einer dem hohen Alter des Patienten entsprechenden Arterio. sklerose und einer infolge der durchgemachten Pneumonie bestehen den Dyspnoe begründet sein. Ferner ist die geradezu glänzende Einwirkung des Silbersalvarsans auf Eiweißmenge, spezifisches Gewicht und Oedeme zahlenmäBig zu verfolgen und entspricht (wie es sich ja auch Kolle im Tierversuch als doppelt bis dreimal so wirksam als das Altsalvarsan erwiesen hatte) in seiner raschen, innerhalb von zehn Tagen zum völligen Schwinden der Eiweißausscheidung führenden Wirkung den mannigfachen Berichten über prompte Einwirkung auf frische syphilitische Erscheinungen und ihren Spirochätengehalt

Nicht aber erstreckt sich diese gute Wirkung auf den Ablauf der Wa.R. Trotz späterer Kombination mit $\mathrm{Hg}$ ist die Wa.R. am 18. VI. nach 20tägiger, allerdings leichter Schmierkur (mit $21 / 3$ täglich) und nach 12 Silbersalvarsaninjektionen bei der doch frischen 
Lues II immer noch positiv. Erst nach 3 weiteren Injektionen von je 0.45 Salvarsannatrium und verstärkter Schmierkur wurde die Wa.R. endlich negativ. Ob weitere Gaben von Silbersalvarsan allein dieselbe Wirkung gehabt hätten, muß dahingestellt bleiben. Unbestritten bleibt jedenfalls bei unserer Beobachtung die gute Einwirkung des Silbersalvarsans auf die luetische Nephrose. Wenn auch bisher dic Anwendung der kombinierten Kur in derartiges Fällen zu guten Erfolgen führte, wie Wertheim ${ }^{2}$ ) aus unserer Klinik bezúglich Kombination von Salvarsan mit leichter Schmierkur berichten konnte und wie in einer weiteren Dissertation demnächst für Anwendung von Salvarsannatrium mit Schmierkur nachgewiesen werden soll, und wenn auch Buschke ferner in derartigen Fällen mit kleinen Dosen Salizylquecksilber allein zum Ziele kam, so dürfte doch eine Behandlung, welche wenigstens zu Anfang den geschädigten Nierenepithelien das vielseitig als Protoplasmagift angesprochene Queck. silber nicht zumutet und eine energische, schnellwirkende spirochätozide Wirkung entfaltet, sicher Beachtung verdienen.

Unsere bisherigen Erfahrungen bei der Behandlung von Lues II in Verein mit den oben erwähnten Angaben der Literatur haben uns veranlaBt, speziell auch zwecks besserer Beeinflussung der Wa.R. nach dem Schwinden der Nierenerscheinungen $\mathrm{Hg}$ zu Hilfe $\mathrm{zu}$ thelumen.

Besonders bei Lues Il dürften der Salvarsan- und, wie sich aus allen Veröffentlichungen immer wieder zeigt, auch der Silbersalvarsan. therapie durch die von $E$. $H$ of $f m$ an $n$ stets verfochtene kombinierte simultane Kur aluf die Datuer mehr Anhänger erworben werden, als die erste Begeisterung über die reine Silbersalvarsantherapie es wohl vermag. Auch wir sehen in dem stark spirochätoziden Mittel des Silbersalvarsans eine wertvolle Bereicherung unseres chemotherapeutischen Schatzes; eine bisher kaum beachtete besondere In. dikation zu seiner Anwendung sollte im Vorstehenden hervorgehoben werden: Auch bei Nephrose wird Silbersalvarsan gut vertragen, erweist sich als durchaus brauchbar, wenn auch die Wa.R. langsam beeinflubt wurde. 